\title{
31. GEOPHYSICAL DATA COLLECTED UNDERWAY ON GLOMAR CHALLENGER, LEG 47B'
}

\author{
Jean-Claude Sibuet, Centre Oceanologique de Bretagne, B.P. 337, 29273 Brest, France \\ William B.F. Ryan, Lamont-Doherty Geological Observatory, Palisades, New York \\ and
}

Ted B. Gustafson and S.M. Smith, Scripps Institution of Oceanography, La Jolla, California

The scientific party aboard Glomar Challenger collected geophysical data during DSDP Leg 47B, which commenced from Vigo, Spain, and terminated at Brest, France (Figure 1). Site $398\left(40^{\circ} 57.6^{\prime} \mathrm{N}, 10^{\circ} 43.1^{\prime} \mathrm{W}\right)$, located just south of Vigo Seamount and about 95 miles west of Porto, Portugal, was drilled from 13 April 1976 to 9 May 1976. Table 1 gives satellite positions, distance (in nautical miles along the track) and the speed and course between successive navigation points. The positioning error along the trackline is approximately 1 nautical mile. Distances are shown (Figure 1) along the trackline every 50 nautical miles and annotated every 100 nautical miles. Time is marked by crosses every 2 hours and annotated every 4 hours.

Figure 2 shows the magnetic anomaly and bathymetric data plotted as functions of time, distance, latitude, and longitude (with distance plotted as a linear function). Dates and positions of major course changes ( $>30$ degrees) are annotated. Magnetic anomaly values have been computed using the 1965 IGRF regional field; depth is expressed in uncorrected meters assuming a sound velocity of $1500 \mathrm{~m} / \mathrm{s}$. The site location and significant bathymetric features also are annotated on the profile. Sections of track having sub-bottom profiler records are represented by a solid line along the bottom of the profile.

Figures 3 to 8 are the seismic reflection records shown as a linear function of time. Profiles are annotated every hour and at each change of course and speed. Depth is annotated on the sides of the profiles in seconds of twoway travel time. The sound source for these data was two bolt airguns with 10 and $40 \mathrm{in}^{3}$ firing chambers. The data were recorded on dry-paper EDO recorders employing 10 and 5 -s sweep rate after filtering frequencies outside the 40 to $320 \mathrm{~Hz}$ range.

${ }^{1}$ Contribution 583 of the Scientific Department of the Centre Oceanologique de Bretagne.

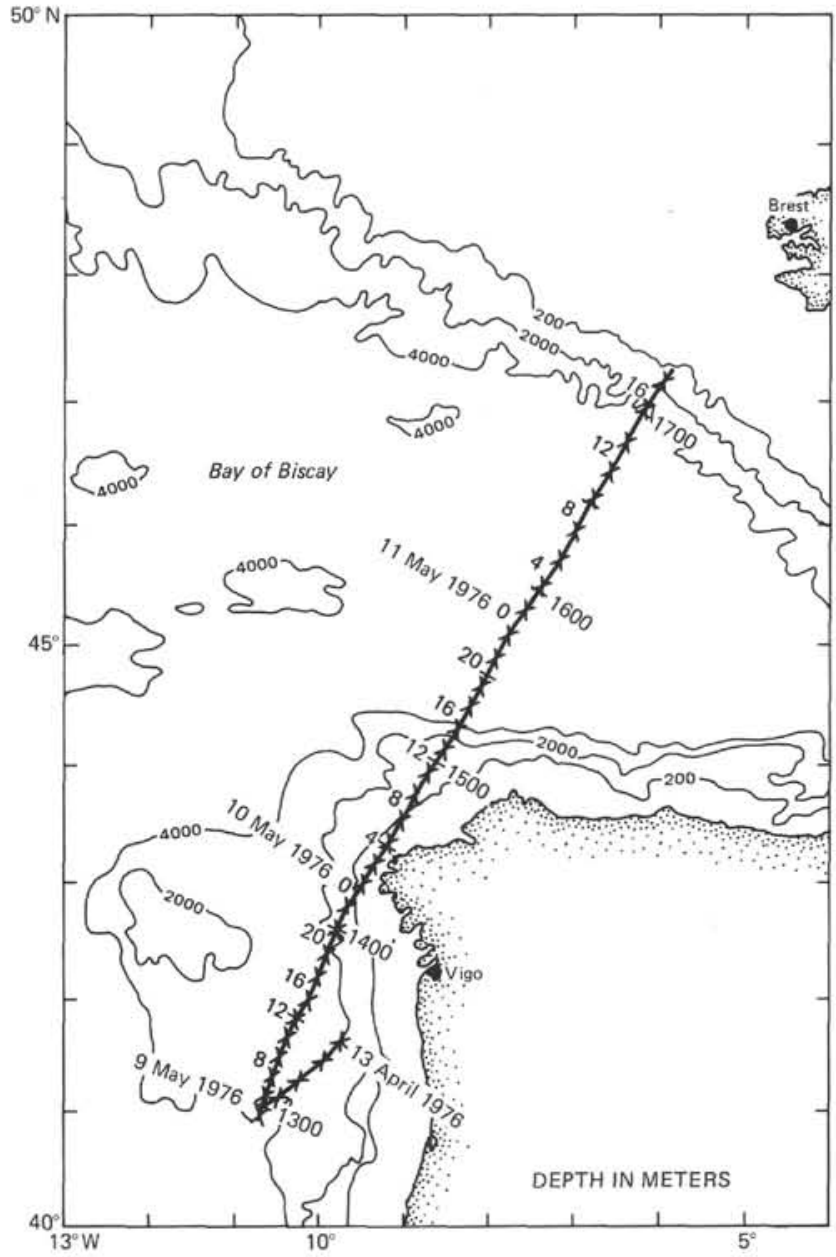

Figure 1. Leg 47B navigation, Mercator projection.

\section{ACKNOWLEDGMENTS}

We especially acknowledge the DSDP technical staff onboard Glomar Challenger during Leg 47B for their valuable assistance and competence during this cruise. The processed data have been prepared by the Underway Data Processing Group, SIO and the Geological Data Center of Scripps Institution of Oceanography. 
TABLE 1

Navigation, Leg 47B (1976)

\begin{tabular}{|c|c|c|c|c|c|c|c|c|c|}
\hline Day & Month & $\begin{array}{l}\text { Time } \\
\text { Zone }\end{array}$ & Hour & $\begin{array}{l}\text { Latitude } \\
\text { (N) }\end{array}$ & $\begin{array}{l}\text { Longitude } \\
\text { (W) }\end{array}$ & $\begin{array}{l}\text { Distance } \\
\text { (n. mi.) }\end{array}$ & $\begin{array}{l}\text { Speed } \\
\text { (knots) }\end{array}$ & $\begin{array}{c}\text { Course } \\
\left({ }^{\circ}\right)\end{array}$ & $\begin{array}{l}\text { Nav. } \\
\text { Points }\end{array}$ \\
\hline 13 & 4 & 0 & 956 & $41^{\circ} 39.4^{\prime}$ & $9^{\circ} 42.8^{\prime}$ & 1229.1 & 8.5 & 222 & 1 \\
\hline 13 & 4 & 0 & 1032 & $41^{\circ} 35.6^{\prime}$ & $9^{\circ} 47.4^{\prime}$ & 1234.2 & 6.1 & 214 & 2 \\
\hline 13 & 4 & 0 & 1045 & $41^{\circ} 34.5^{\prime}$ & $9^{\circ} 48.4^{\prime}$ & 1235.5 & 8.1 & 222 & 3 \\
\hline 13 & 4 & 0 & 1050 & $41^{\circ} 34.0^{\prime}$ & $9^{\circ} 49.0^{\prime}$ & 1236.2 & 5.0 & 218 & 4 \\
\hline 13 & 4 & 0 & 1128 & $41^{\circ} 31.5^{\prime}$ & $9^{\circ} 51.6^{\prime}$ & 1239.3 & 8.3 & 232 & 5 \\
\hline 13 & 4 & 0 & 1312 & $41^{\circ} 22.5^{\prime}$ & $10^{\circ} 6.7^{\prime}$ & 1253.8 & 8.4 & 232 & 6 \\
\hline 13 & 4 & 0 & 1502 & $41^{\circ} 12.9^{\prime}$ & $10^{\circ} 22.8^{\prime}$ & 1269.2 & 8.5 & 233 & 7 \\
\hline 13 & 4 & 0 & 1620 & $41^{\circ} 6.3^{\prime}$ & $10^{\circ} 34.5^{\prime}$ & 1280.3 & 7.9 & 231 & 8 \\
\hline 13 & 4 & 0 & 1645 & $41^{\circ} 4.2^{\prime}$ & $10^{\circ} 37.9^{\prime}$ & 1283.6 & 8.9 & 198 & 9 \\
\hline 13 & 4 & 0 & 1650 & $41^{\circ} 3.5^{\prime}$ & $10^{\circ} 38.2^{\prime}$ & 1284.3 & 9.3 & 212 & 10 \\
\hline 13 & 4 & 0 & 1729 & $40^{\circ} 58.4^{\prime}$ & $10^{\circ} 42.5^{\prime}$ & 1290.3 & 7.6 & 207 & 11 \\
\hline 13 & 4 & 0 & 1737 & $40^{\circ} 57.5^{\prime}$ & $10^{\circ} 43.1^{\prime}$ & 1291.4 & 0.0 & 90 & 12 \\
\hline 9 & 5 & 0 & 000 & $40^{\circ} 57.5^{\prime}$ & $10^{\circ} 43.1^{\prime}$ & 1291.4 & 0.3 & 63 & 13 \\
\hline 9 & 5 & 0 & 324 & $40^{\circ} 58.0^{\prime}$ & $10^{\circ} 41.8^{\prime}$ & 1292.5 & 4.1 & 191 & 14 \\
\hline 9 & 5 & 0 & 330 & $40^{\circ} 57.6^{\prime}$ & $10^{\circ} 41.9^{\prime}$ & 1292.9 & 4.5 & 37 & 15 \\
\hline 9 & 5 & 0 & 335 & $40^{\circ} 57.9^{\prime}$ & $10^{\circ} 41.6^{\prime}$ & 1293.2 & 4.9 & 10 & 16 \\
\hline 9 & 5 & 0 & 412 & $41^{\circ} 0.9^{\prime}$ & $10^{\circ} 40.9^{\prime}$ & 1296.3 & 5.0 & 6 & 17 \\
\hline 9 & 5 & 0 & 430 & $41^{\circ} 2.4^{\prime}$ & $10^{\circ} 40.7^{\prime}$ & 1297.8 & 4.2 & 8 & 18 \\
\hline 9 & 5 & 0 & 446 & $41^{\circ} 3.5^{\prime}$ & $10^{\circ} 40.5^{\prime}$ & 1298.9 & 5.6 & 24 & 19 \\
\hline 9 & 5 & 0 & 500 & $41^{\circ} 4.7^{\prime}$ & $10^{\circ} 39.8^{\circ}$ & 1300.2 & 5.5 & 24 & 20 \\
\hline 9 & 5 & 0 & 512 & $41^{\circ} 5.7^{\prime}$ & $10^{\circ} 39.2^{\prime}$ & 1301.3 & 5.8 & 18 & 21 \\
\hline 9 & 5 & 0 & 600 & $41^{\circ} 10.1^{\prime}$ & $10^{\circ} 37.3^{\prime}$ & 1305.9 & 5.8 & 16 & 22 \\
\hline 9 & 5 & 0 & 630 & $41^{\circ} 12.9^{\prime}$ & $10^{\circ} 36.2^{\prime}$ & 1308.9 & 5.4 & 21 & 23 \\
\hline 9 & 5 & 0 & 915 & $41^{\circ} 26.8^{\prime}$ & $10^{\circ} 29.1^{\prime}$ & 1323.7 & 5.5 & 23 & 24 \\
\hline 9 & 5 & 0 & 1222 & $41^{\circ} 42.5^{\prime}$ & $10^{\circ} 20.4^{\prime}$ & 1340.7 & 5.7 & 19 & 25 \\
\hline 9 & 5 & 0 & 1300 & $41^{\circ} 45.9^{\prime}$ & $10^{\circ} 18.8^{\prime}$ & 1344.3 & 5.8 & 28 & 26 \\
\hline 9 & 5 & 0 & 1334 & $41^{\circ} 48.8^{\prime}$ & $10^{\circ} 16.7^{\prime}$ & 1347.6 & 5.7 & 28 & 27 \\
\hline 9 & 5 & 0 & 1434 & $41^{\circ} 53.8^{\prime}$ & $10^{\circ} 13.1^{\prime}$ & 1353.3 & 5.8 & 29 & 28 \\
\hline 9 & 5 & 0 & 1520 & $41^{\circ} 57.7^{\prime}$ & $10^{\circ} 10.2^{\prime}$ & 1357.8 & 6.0 & 30 & 29 \\
\hline 9 & 5 & 0 & 1714 & $42^{\circ} 7.6^{\circ}$ & $10^{\circ} 2.6^{\prime}$ & 1369.2 & 6.3 & 24 & 30 \\
\hline 9 & 5 & 0 & 1735 & $42^{\circ} 9.6^{\prime}$ & $10^{\circ} 1.4^{\prime}$ & 1371.4 & 6.1 & 22 & 31 \\
\hline 9 & 5 & 0 & 1930 & $42^{\circ} 20.5^{\prime}$ & $9^{\circ} 55.4^{\prime}$ & 1383.1 & 6.7 & 19 & 32 \\
\hline 9 & 5 & 0 & 2030 & $42^{\circ} 26.8^{\prime}$ & $9^{\circ} 52.5^{\prime}$ & 1389.8 & 6.6 & 22 & 33 \\
\hline 9 & 5 & 0 & 2320 & $42^{\circ} 44.3^{\prime}$ & $9^{\circ} 43.1^{\prime}$ & 1408.6 & 6.5 & 36 & 34 \\
\hline 9 & 5 & 0 & 2328 & $42^{\circ} 45.0^{\circ}$ & $9^{\circ} 42.4^{\prime}$ & 1409.5 & 6.8 & 34 & 35 \\
\hline 10 & 5 & 0 & 000 & $42^{\circ} 48.0^{\prime}$ & $9^{\circ} 39.6^{\prime}$ & 1413.1 & 6.8 & 34 & 36 \\
\hline 10 & 5 & 0 & 100 & $42^{\circ} 53.6^{\circ}$ & $9^{\circ} 34.4^{\prime}$ & 1419.9 & 6.7 & 27 & 37 \\
\hline 10 & 5 & 0 & 136 & $42^{\circ} 57.2^{\prime}$ & $9^{\circ} 31.9^{\prime}$ & 1423.9 & 6.8 & 28 & 38 \\
\hline 10 & 5 & 0 & 215 & $43^{\circ} 1.1^{\prime}$ & $9^{\circ} 29.1^{\prime}$ & 1428.3 & 6.7 & 33 & 39 \\
\hline 10 & 5 & 0 & 322 & $43^{\circ} 7.4^{\prime}$ & $9^{\circ} 23.6^{\prime}$ & 1435.8 & 6.3 & 37 & 40 \\
\hline 10 & 5 & 0 & 422 & $43^{\circ} 12.4^{\prime}$ & $9^{\circ} 18.4^{\prime}$ & 1442.1 & 6.6 & 36 & 41 \\
\hline 10 & 5 & 0 & 440 & $43^{\circ} 14.0^{\prime}$ & $9^{\circ} 16.8^{\prime}$ & 1444.0 & 7.0 & 39 & 42 \\
\hline 10 & 5 & 0 & 450 & $43^{\circ} 14.9^{\prime}$ & $9^{\circ} 15.8^{\prime}$ & 1445.2 & 6.4 & 32 & 43 \\
\hline 10 & 5 & 0 & 636 & $43^{\circ} 24.5^{\prime}$ & $9^{\circ} 7.7^{\prime}$ & 1456.5 & 6.7 & 30 & 44 \\
\hline 10 & 5 & 0 & 702 & $43^{\circ} 27.0^{\prime}$ & $9^{\circ} 5.7^{\prime}$ & 1459.4 & 6.9 & 30 & 45 \\
\hline 10 & 5 & 0 & 730 & $43^{\circ} 29.8^{\prime}$ & $9^{\circ} 3.5^{\prime}$ & 1462.6 & 7.0 & 27 & 46 \\
\hline 10 & 5 & 0 & 800 & $43^{\circ} 32.9^{\prime}$ & $9^{\circ} 1.3^{\prime}$ & 1466.1 & 6.9 & 30 & 47 \\
\hline 10 & 5 & 0 & 944 & $43^{\circ} 43.3^{\prime}$ & $8^{\circ} 53.0^{\prime}$ & 1478.1 & 7.1 & 32 & 48 \\
\hline 10 & 5 & 0 & 1126 & $43^{\circ} 53.5^{\prime}$ & $8^{\circ} 44.2^{\prime}$ & 1490.1 & 6.8 & 28 & 49 \\
\hline 10 & 5 & 0 & 1145 & $43^{\circ} 55.4^{\prime}$ & $8^{\circ} 42.8^{\prime}$ & 1492.2 & 6.6 & 29 & 50 \\
\hline 10 & 5 & 0 & 1244 & $44^{\circ} 1.1^{\prime}$ & $8^{\circ} 38.5^{\prime}$ & 1498.7 & 6.4 & 29 & 51 \\
\hline 10 & 5 & 0 & 1315 & $44^{\circ} 4.0^{\prime}$ & $8^{\circ} 36.3^{\prime}$ & 1502.0 & 6.1 & 34 & 52 \\
\hline 10 & 5 & 0 & 1334 & $44^{\circ} 5.6^{\prime}$ & $8^{\circ} 34.8^{\prime}$ & 1504.0 & 6.7 & 34 & 53 \\
\hline 10 & 5 & 0 & 1430 & $44^{\circ} 10.8^{\prime}$ & $8^{\circ} 29.9^{\prime}$ & 1510.2 & 6.7 & 34 & 54 \\
\hline 10 & 5 & 0 & 1600 & $44^{\circ} 19.1^{\prime}$ & $8^{\circ} 22.1^{\prime}$ & 1520.2 & 6.3 & 35 & 55 \\
\hline 10 & 5 & 0 & 1644 & $44^{\circ} 22.9^{\prime}$ & $8^{\circ} 18.4^{\prime}$ & 1524.9 & 6.0 & 31 & 56 \\
\hline 10 & 5 & 0 & 1705 & $44^{\circ} 24.7^{\prime}$ & $8^{\circ} 16.9^{\prime}$ & 1527.0 & 6.1 & 27 & 57 \\
\hline 10 & 5 & 0 & 1750 & $44^{\circ} 28.8^{\prime}$ & $8^{\circ} 14.0^{\prime}$ & 1531.6 & 6.4 & 27 & 58 \\
\hline 10 & 5 & 0 & 1830 & $44^{\circ} 32.6^{\prime}$ & $8^{\circ} 11.3^{\prime}$ & 1535.8 & 6.5 & 29 & 59 \\
\hline 10 & 5 & 0 & 1956 & $44^{\circ} 40.7^{\prime}$ & $8^{\circ} 4.9^{\prime}$ & 1545.1 & 6.9 & 28 & 60 \\
\hline 10 & 5 & 0 & 2232 & $44^{\circ} 56.5^{\prime}$ & $7^{\circ} 53.3^{\prime}$ & 1562.9 & 6.9 & 24 & 61 \\
\hline 10 & 5 & 0 & 2330 & $45^{\circ} 2.6^{\prime}$ & $7^{\circ} 49.4^{\prime}$ & 1569.6 & 6.9 & 29 & 62 \\
\hline 11 & 5 & 0 & 000 & $45^{\circ} 5.6^{\prime}$ & $7^{\circ} 47.0^{\prime}$ & 1573.1 & 6.9 & 29 & 63 \\
\hline 11 & 5 & 0 & 020 & $45^{\circ} 7.6^{\prime}$ & $7^{\circ} 45.4^{\prime}$ & 1575.4 & 7.4 & 29 & 64 \\
\hline 11 & 5 & 0 & 100 & $45^{\circ} 11.9^{\prime}$ & $7^{\circ} 42.0^{\prime}$ & 1580.3 & 7.3 & 33 & 65 \\
\hline 11 & 5 & 0 & 148 & $45^{\circ} 16.8^{\prime}$ & $7^{\circ} 37.4^{\prime}$ & 1586.2 & 7.3 & 35 & 66 \\
\hline 11 & 5 & 0 & 234 & $45^{\circ} 21.4^{\prime}$ & $7^{\circ} 32.8^{\prime}$ & 1591.8 & 7.8 & 36 & 67 \\
\hline 11 & 5 & 0 & 454 & $45^{\circ} 36.1^{\prime}$ & $7^{\circ} 17.7^{\prime}$ & 1609.9 & 8.0 & 39 & 68 \\
\hline 11 & 5 & 0 & 520 & $45^{\circ} 38.8^{\prime}$ & $7^{\circ} 14.6^{\prime}$ & 1613.4 & 7.4 & 35 & 69 \\
\hline 11 & 5 & 0 & 522 & $45^{\circ} 39.0^{\prime}$ & $7^{\circ} 14.4^{\prime}$ & 1613.6 & 8.0 & 31 & 70 \\
\hline 11 & 5 & 0 & 642 & $45^{\circ} 48.1^{\prime}$ & $7^{\circ} 6.5^{\prime}$ & 1624.3 & 7.7 & 24 & 71 \\
\hline 11 & 5 & 0 & 716 & $45^{\circ} 52.1^{\prime}$ & $7^{\circ} 4.0^{\prime}$ & 1628.6 & 8.3 & 28 & 72 \\
\hline 11 & 5 & 0 & 1032 & $46^{\circ} 15.8^{\prime}$ & $6^{\circ} 45.5^{\prime}$ & 1655.6 & 8.8 & 35 & 73 \\
\hline 11 & 5 & 0 & 1156 & $46^{\circ} 25.9^{\prime}$ & $6^{\circ} 35.3^{\prime}$ & 1667.9 & 8.8 & 32 & 74 \\
\hline 11 & 5 & 0 & 1220 & $46^{\circ} 28.9^{\prime}$ & $6^{\circ} 32.6^{\prime}$ & 1671.4 & 8.6 & 28 & 75 \\
\hline 11 & 5 & 0 & 1440 & $46^{\circ} 46.6^{\prime}$ & $6^{\circ} 18.7^{\prime}$ & 1691.5 & 8.6 & 29 & 76 \\
\hline 11 & 5 & 0 & 1602 & $46^{\circ} 56.8^{\prime}$ & $6^{\circ} 10.3^{\prime}$ & 1703.2 & 8.3 & 33 & 77 \\
\hline 11 & 5 & 0 & 1628 & $46^{\circ} 59.8^{\prime}$ & $6^{\circ} 7.4^{\prime}$ & 1706.8 & 8.0 & 32 & 78 \\
\hline 11 & 5 & 0 & 1716 & $47^{\circ} 5.2^{\prime}$ & $6^{\circ} 2.4^{\prime}$ & 1713.2 & 7.5 & 33 & 79 \\
\hline 11 & 5 & 0 & 1900 & $47^{\circ} 16.0^{\prime}$ & $5^{\circ} 51.9^{\prime}$ & 1726.1 & 7.5 & 33 & 80 \\
\hline
\end{tabular}



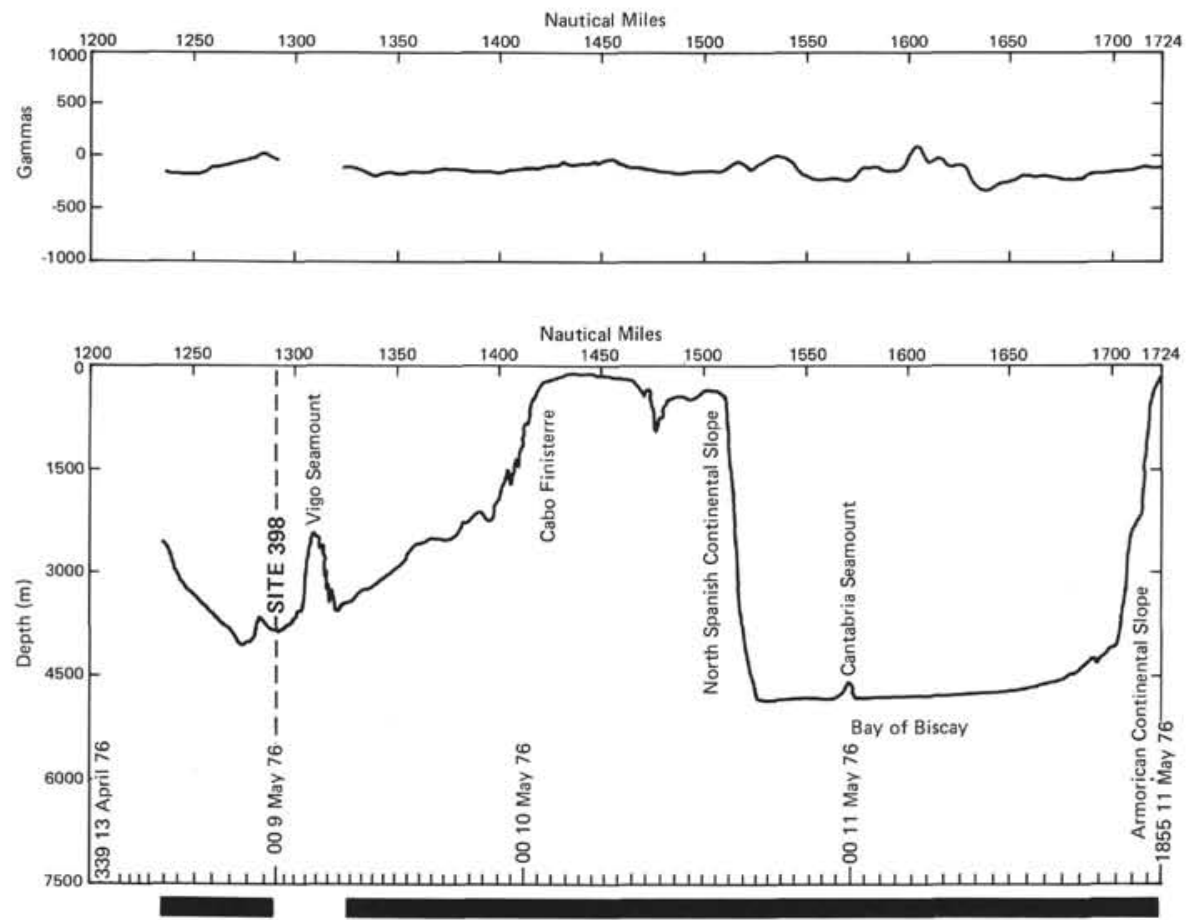

Figure 2. Magnetic and bathymetric profiles, Leg 47B. 


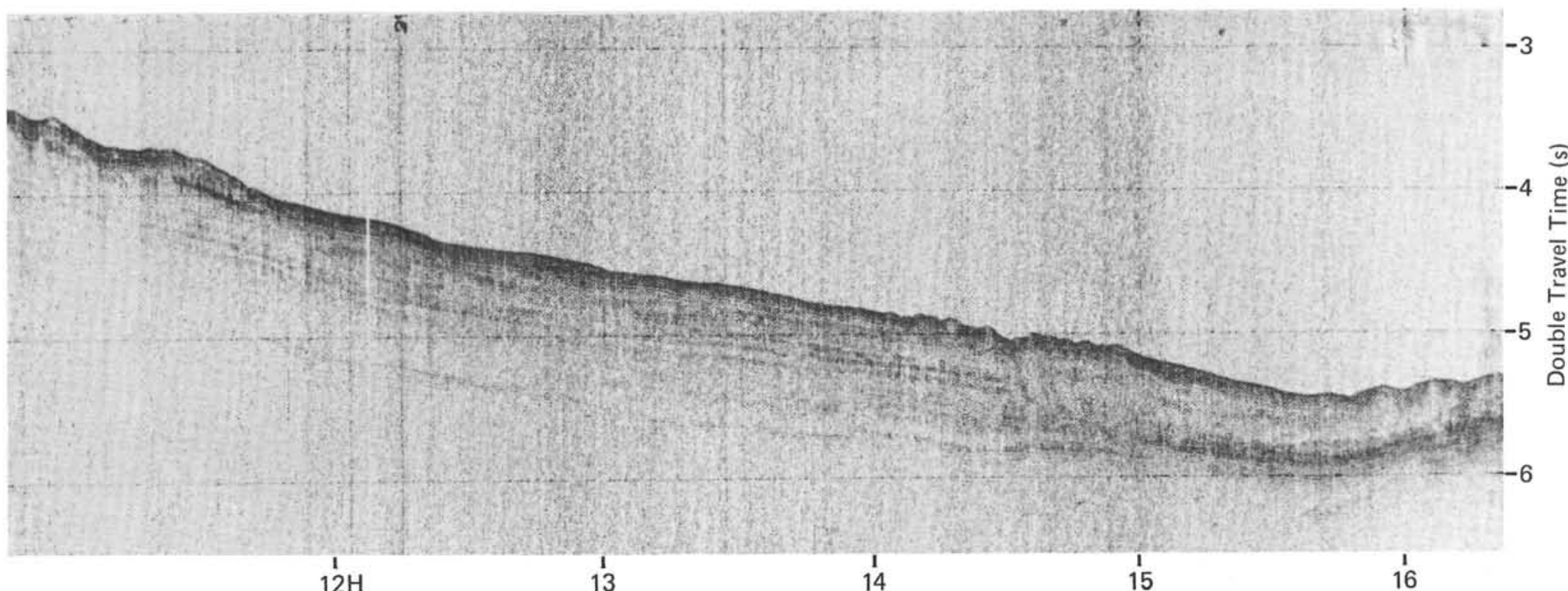

13 APRIL 1976

Site 398

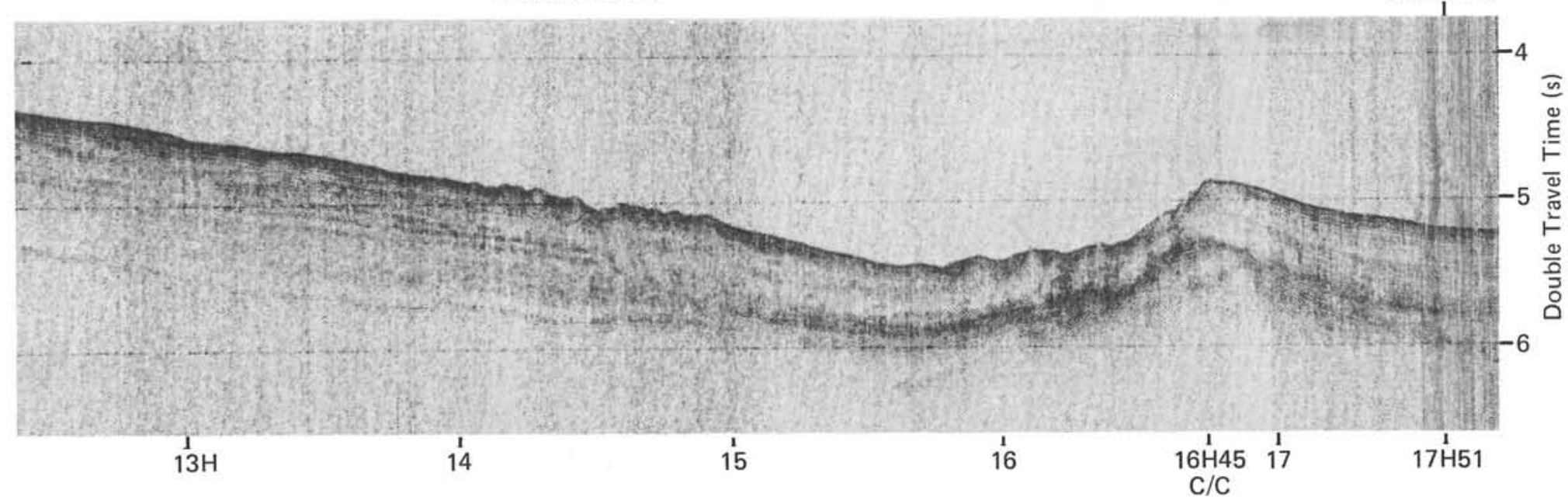

Figure 3. Seismic reflection profiles, Leg $47 B$. 

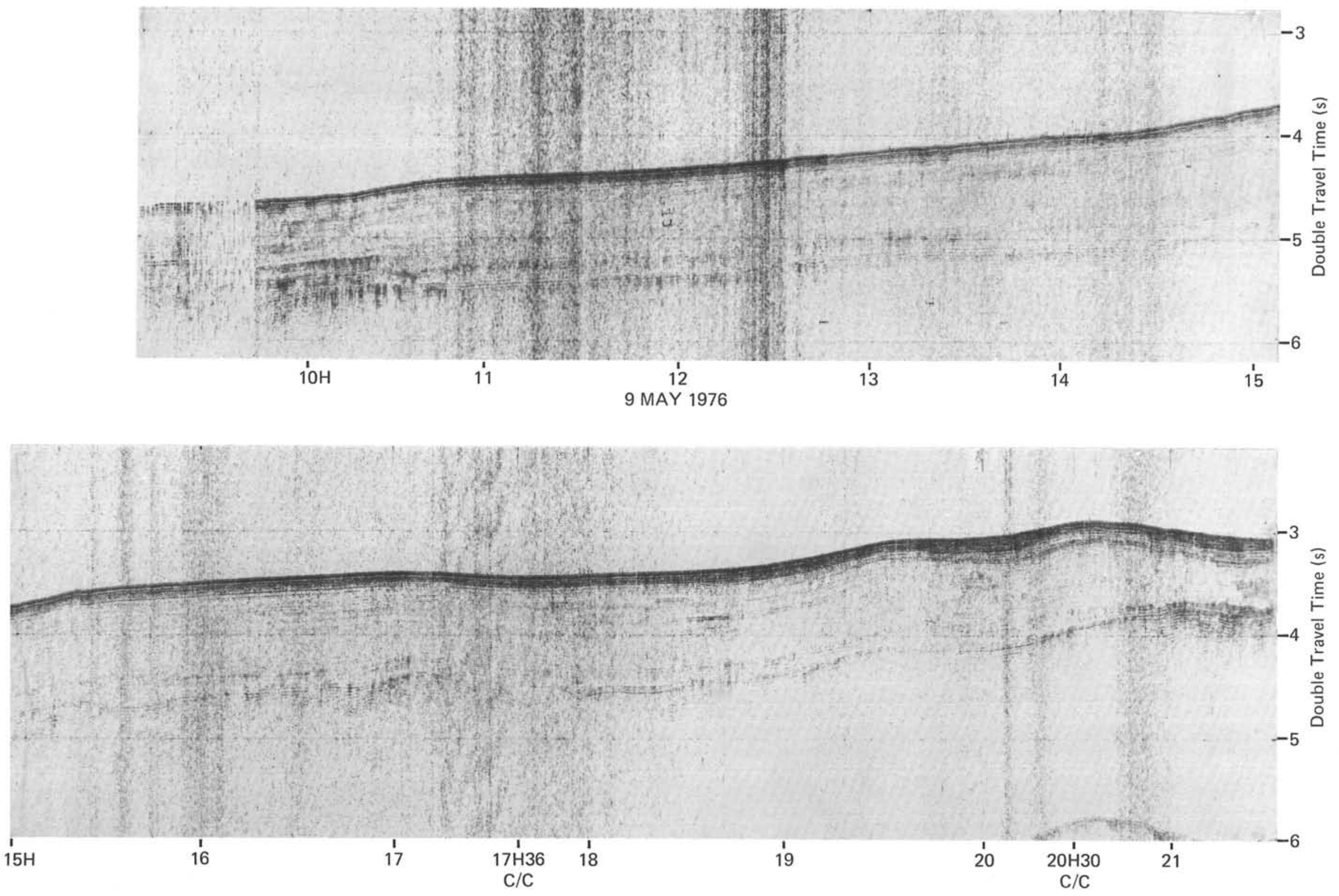

Figure 4. Seismic reflection profiles, Leg 47B. 

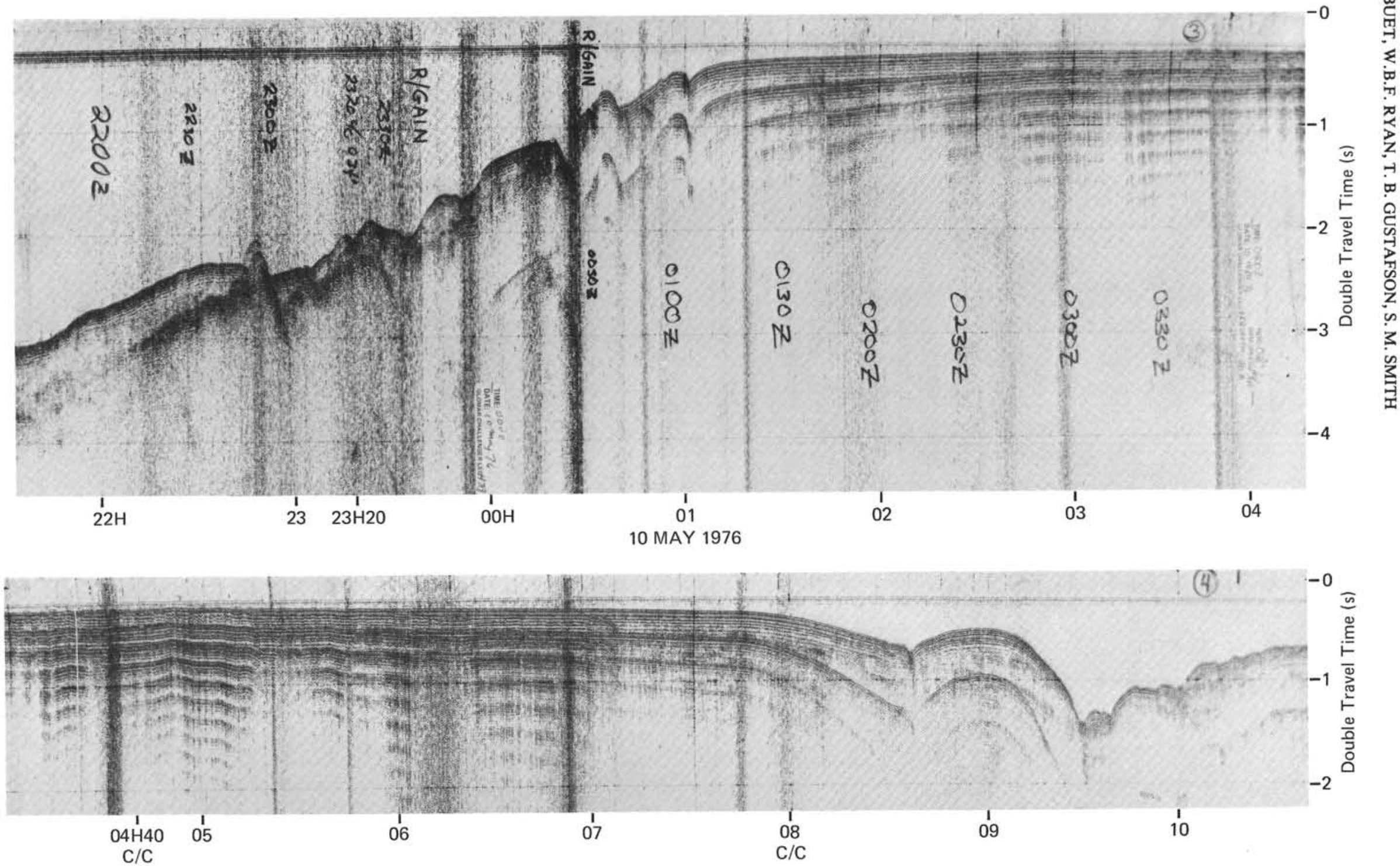

Figure 5. Seismic reflection profiles, Leg $47 B$. 


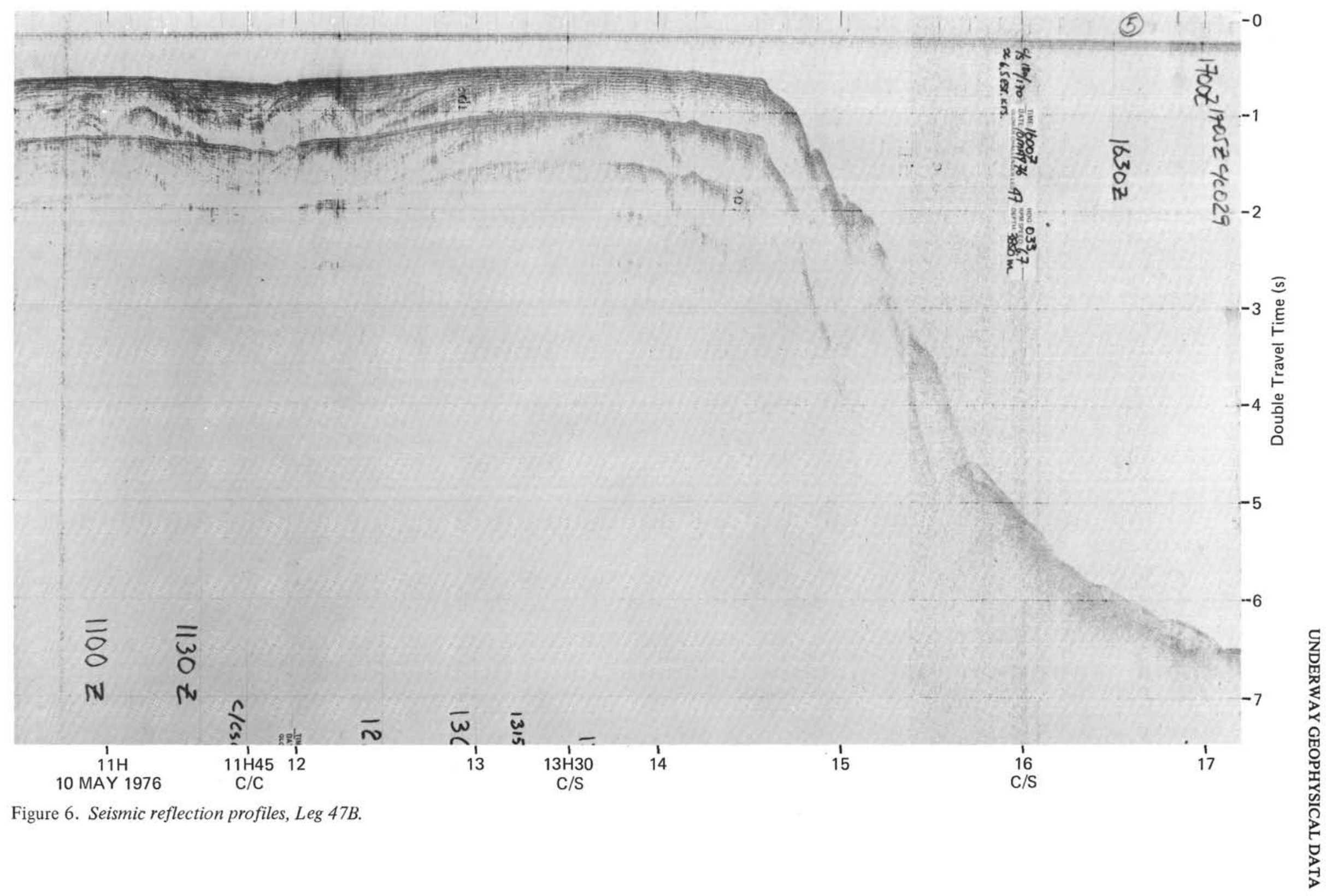



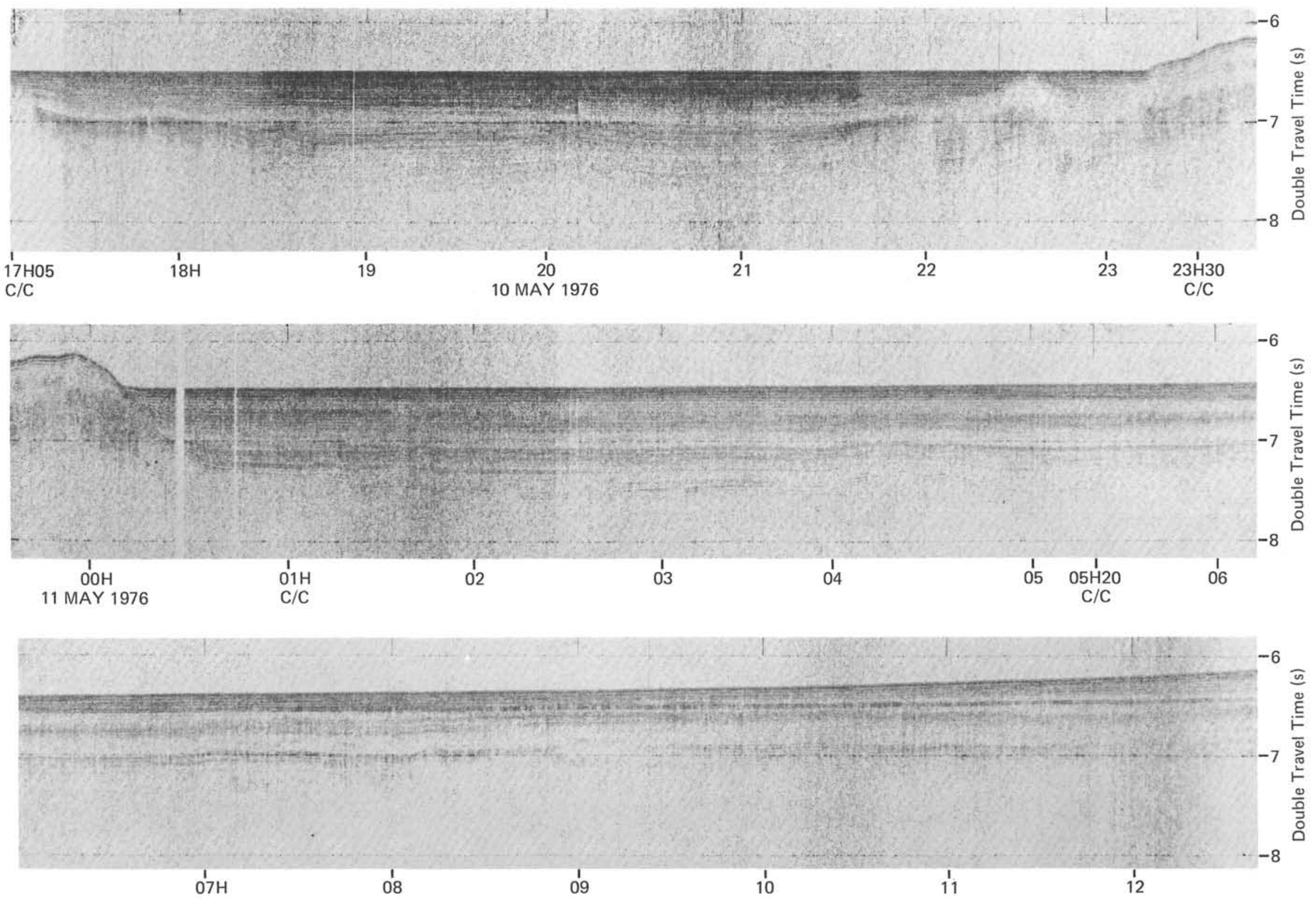

Figure 7. Seismic reflection profiles, Leg $47 B$. 


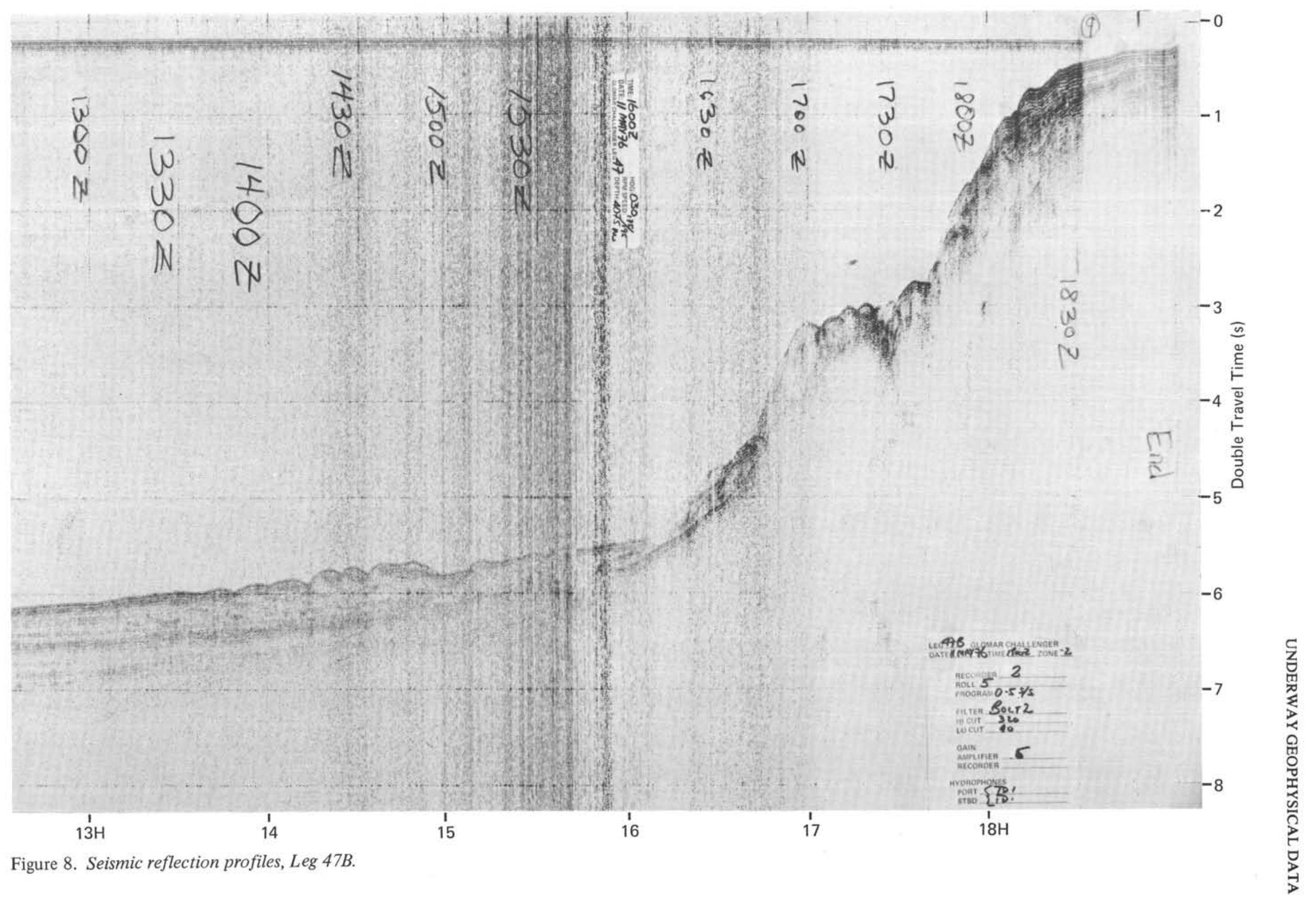

